OPEN ACCESS

Citation: L. Fabbri, F. Bracci (2021) Pratiche di interculturalismo quotidiano. Etnografia di un condominio multietnico. Rief 18, 2: pp. 107-119. doi: https://doi. org/10.36253/rief-10606.

Copyright: (c) 2021 E. Pelizzari. This is an open access, peer-reviewed article published by Firenze University Press (https:// oaj.fupress.net/index.php/rief) and distributed under the terms of the Creative Commons Attribution License, which permits unrestricted use, distribution, and reproduction in any medium, provided the original author and source are credited.

Data Availability Statement: All relevant data are within the paper and its Supporting Information files.

Competing Interests: The Author(s) declare(s) no conflict of interest.

\section{Pratiche di interculturalismo quotidiano. Etnografia di un condominio multietnico}

\author{
Loretta Fabbri ${ }^{1}$, Francesca Bracci ${ }^{2,3}$
}

Abstract

L'articolo esplora i processi di costruzione della differenza nella materialità di uno spazio di interculturalismo quotidiano, attraverso l'analisi delle interazioni che scandiscono la vita di un condominio multietnico situato nel centro storico di Arezzo. È stato condotto uno studio etnografico che - inserendosi nel movimento più ampio delle metodologie post-qualitative - ha inglobato nel processo di analisi le nostre esperienze e interpretazioni personali in quanto partecipi della realtà osservata come residenti nel complesso abitativo. L'interesse è rivolto allo studio dell'uso pratico della differenza in contesti di interazione quotidiana, esaminando un condominio quale spazio effettivo di convivenza multietnica dove materia, soggetti, spazi, categorie e geografie si articolano lungo un continuum di senso ed esperienza, che ha trovato nel palazzo e nelle sue "pratiche dell'abitare" un laboratorio di apprendimento.

Parole chiave: interculturalismo quotidiano, apprendimento informale, etnografia, differenza.

\section{Abstract}

This paper explores the processes of construction of difference in the materiality of a space of daily interculturalism, through the analysis of the interactions characterizing the life of a multiethnic condominium, which is located in the historic center of Arezzo (Tuscany, Italy). An ethnographic study was conducted which - entering the wider movement of post-qualitative methodologies - incorporated in the analysis process our experiences and personal interpretations, since directly involved and participating in the reality observed as residents in the housing complex. Our interest is aimed at studying the practical use of difference in everyday life, analyzing a condominium as effective space of multiethnic coexistence where matter, subjects, spaces, categories, and geog-

${ }^{1}$ Professoressa ordinaria di Didattica e Pedagogia speciale presso il Dipartimento di Scienze della Formazione, Scienze umane e della Comunicazione interculturale dell'Università degli Studi di Siena.

${ }^{2}$ Professoressa associata di Didattica e Pedagogia speciale presso il Dipartimento di Formazione, Lingue, Intercultura, Letterature e Psicologia (FORLILPSI) dell'Università degli Studi di Firenze.

${ }^{3}$ Sono da attribuire a Loretta Fabbri i paragrafi 3, 4 e le Considerazioni conclusive. Sono invece da attribuire a Francesca Bracci l'Introduzione e i paragrafi 1 e 2 (d'ora in avanti, laddove non diversamente specificato, le note a piè di pagina sono da intendersi a cura delle Autrici, N.d.R.). 
raphies are articulated along a continuum of sense and experience, which has found a learning laboratory in the building and its "practices of living".

Keywords: daily interculturalism, informal learning, ethnography, difference.

\title{
Introduzione
}

\author{
Quando rifutiamo l'unica storia, \\ quando ci rendiamo conto che non c’è mai \\ un'unica storia per nessun luogo, \\ riconquistiamo una sorta di paradiso. \\ Ngozi Adichie (2016, trad. it. 2020, p. 9)
}

L'articolo esplora i processi di costruzione della differenza nella materialità di uno spazio di interculturalismo quotidiano, attraverso l'analisi delle interazioni che scandiscono la vita di un condominio multietnico situato nel centro storico di Arezzo. L'obiettivo è contribuire a rispondere a questa domanda: come è possibile sopravvivere in contesti caratterizzati dall' incontro quotidiano con la differenza? Come è possibile sopravvivere quando gli stranieri diventano vicini (Sandercock, 2000)? Due quesiti che rappresentano modi per declinare il noto interrogativo di Touraine: "pourrons-nous vivre ensemble?» (1997, passim).

È stato condotto uno studio etnografico che - inserendosi nel movimento più ampio delle metodologie post-qualitative (Fabbri, 2019; Gherardi, 2017; St. Pierre, 2015) - ha inglobato nel processo di analisi le nostre esperienze e interpretazioni personali, in quanto direttamente partecipi della realtà osservata, in quanto residenti nel condominio. Come Ricercatrici, non abbiamo inteso essere delle "osservatrici privilegiate"; piuttosto, il nostro ruolo è da collocarsi nei contesti di azione e nei processi interattivi in atto al loro interno. Il lavoro etnografico ha avuto una durata di due anni, da quando una di noi è tornata ad abitare stabilmente nel complesso condominiale, ma è intrecciato in modo inestricabile alle nostre storie di vita, che ci vedono residenti dal 1998, quando l'edificio accoglieva solo famiglie autoctone.

La scelta etnografica ci è apparsa come la più appropriata, all'interno di una prospettiva costruzionista che interpreta l'interculturalismo come fenomeno processuale e relazionale (Mantovani, 2006). Di qui, il considerare la differenza una produzione situata: il risultato di confronti e conflitti che hanno luogo in contesti contraddistinti da una distribuzione asimmetrica di risorse e potere e, allo stesso tempo, necessaria per attribuire senso alla realtà sociale e per organizzare l'agire in essa (Colombo, Semi, 2007).

Il nostro interesse è rivolto allo studio dell'uso pratico della differenza in contesti di vita quotidiana, analizzando un condominio quale spazio effettivo di convivenza multietnica dove materia, soggetti, spazi, categorie e geografie si articolano lungo un continuum di senso ed esperienza, che ha trovato nel palazzo e nelle sue pratiche dell'abitare un laboratorio di apprendimento.

L'esperienza presentata, inoltre, stimola una riflessione sugli apprendimenti incidentali (Marsick, Neaman, 2018; Id., Watkins, 2018), autodiretti e trasformativi (Brookfield, 1990, 2003; Mezirow, 2000; 2004, trad. it. 2004); che il percorso personale e di ricerca hanno per- 
messo di conseguire, rendendoci, tra l'altro, maggiormente consapevoli della nostra whiteness - categoria verso la quale rischiamo di avere una cecità culturalmente assimilata ${ }^{4}$ (Crenshaw, 1997; Harris, 1993).

Alla luce di tutto questo, dunque, i prossimi paragrafi affrontano, rispettivamente: (1) le prospettive concettuali e i quadri teorici sottesi al processo di ricerca; (2) le scelte metodologiche intraprese; (3) alcune etnografie raccontate per episodi, quali espedienti narrativi utili a far "parlare" i dati raccolti, attraverso interviste, osservazioni e colloqui informali.

\section{Framework teorico}

L'intensificarsi dei processi di globalizzazione e innovazione tecnologica, la crescita di interconnessioni complesse (Harris, 2009) e il crearsi di sfere pubbliche diasporiche (Meer, Modood, 2012) alimentate da flussi planetari di persone, idee, capitali, merci, informazioni, pongono innumerevoli sfide e dilemmi, tra cui la necessità di trovare nuove forme per organizzare, in società sempre più plurali, il vivere quotidiano con la differenza (Colombo, 2014). Del resto, nella vita quotidiana, l'incontro tra corpi non fluttua nel vuoto ma è mediato da narrazioni, immagini, trame complesse e in divenire, che si fondano su una miscela di storie e memorie ritenute reali e altre immaginarie. Questo è ancora più evidente nei contesti di convivenza multietnica, dove la coabitazione tra mondi sociali differenti è divenuta caratterizzante il vivere urbano (Wirth, 1938). Al loro interno, la percezione di oggetti, eventi, soggetti è data dalla sintesi delle adiacenze spaziali, delle sequenze di relazioni e dei significati che ne determinano il manifestarsi (Mantovani, 2008a).

Attraverso i flussi migratori individui, gruppi e comunità si de-territorializzano e ri-territorializzano, contribuendo a modificare i paesaggi urbani e facendo sì che differenti mappe simboliche tendano a sovrapporsi e intrecciarsi a scale di intensità e livelli di complessità sempre maggiori (Cancellieri, 2010; Colombo, 2006). Analizzare i processi di territorializzazione della differenza non ne comporta l'accettazione incondizionata o la celebrazione, ma significa esplorarne il suo uso attivo, situato e orientato a dare senso alle interazioni e ai contesti di azione - ponendo particolare attenzione allo studio di situazioni di multiculturalismo quotidiano, contraddistinte da relazioni problematiche, forme conflittuali, discriminazioni e semplificazioni cariche di pregiudizi e razzismo (Besozzi, 2001).

È stata adottata una visione costruzionista della differenza, che considera culture, appartenenze e identità non come realtà omogenee e coerenti o nuclei stabili, ma come spazi di scambio, risorse per l'azione, repertori polifonici che possono essere contraddittori e frammentari, condivisi e negoziati o contestati e trasformati (Benhabib, 2002; Mantovani, 2008b).

Allo stesso tempo, la produzione sociale della differenza è da assumere come capace di generare fatti sociali che hanno implicazioni concrete nella vita delle persone, soprattutto quando i loro effetti sono percepiti come stabili, naturali, dati per scontato e indiscussi. Ciò

\footnotetext{
${ }^{4}$ La nozione di whiteness o bianchezza ha origine negli Stati Uniti nell'ambito degli studi intersezionali (Cho, Crenshaw, McCall, 2013; Crenshaw, 1989) ed è inestricabilmente connessa ai costrutti di identità, differenza, diversità e uguaglianza. La whiteness è una categoria interpretativa che ha contribuito a iniziare ad analizzare criticamente le forme contemporanee di dominio e subordinazione, esplorando come il potere sia esercitato in modi che condizionano situazioni di svantaggio e come le fonti di privilegio siano determinate da dimensioni materiali (per esempio, la condizione economica), biologiche (per esempio, il sesso) e giuridiche (per esempio, la cittadinanza). Un aspetto definitorio la whiteness è il privilegio razziale bianco attivo, per troppo tempo rimosso negli ordinamenti e nelle coscienze occidentali.
} 
sollecita a porre in primo piano la necessità di andare oltre la constatazione, più o meno banale, che la differenza sia una costruzione sociale e concentrarsi sui modi attraverso cui questi processi di costruzione prendono forma, analizzando i contesti, micro e macro, che li rendono possibili (Colombo, Semi, 2007). La differenza, quindi, è vista come una pratica, una performance continua, a volte risultato di conflitti manifesti a volte del lavoro di routine orientato a confermare o modificare il senso condiviso attribuito alla realtà in cui si è inseriti (Sarat, 2000). In altri termini, per cogliere la rilevanza assunta dalla differenza in un mondo globale, è utile spostare lo sguardo sulle pratiche quotidiane e analizzare come la differenza e la cultura sono utilizzate nelle interazioni sociali, da chi, in quali contesti, per quali scopi e con quali risultati.

Gli approcci all'interno dei quali abbiamo collocato e definito il concetto di differenza sono riconducibili alla prospettiva interculturale (Benhabib, 2002; Mantovani, 2004, 2006, 2008a; Meer, Modood, 2012) e all'ambito del multiculturalismo quotidiano (Colombo, 2014; Colombo, Semi, 2007). Questi approcci consentono di prendere le distanze dalle teorie multiculturali che suppongono che le società umane siano fondate su una cosa - ovvero, la propria cultura - che separa i membri da quelli di altre società e li rende simili tra loro.

La prospettiva interculturale permette di respingere una concezione di cultura come monolitica, una proprietà di persone e gruppi - come se ogni gruppo umano avesse un qualche tipo di cultura dai contorni ben delineati e facili da descrivere (Benhabib, 2002). La critica che ha mosso al multiculturalismo è di reificare le culture, assumendole come entità separate ed esasperandone sia l'impermeabilità dei confini, sia l'omogeneità interna in termini che possono legittimare richieste repressive di conformità al gruppo. Dall'altra parte, il multiculturalismo quotidiano evidenzia come la concettualizzazione normativa di multiculturalismo - nonostante abbia concorso a rendere centrale la questione dell'eguaglianza delle opportunità e della partecipazione in società in cui la differenza culturale è valorizzata - esaurisca le sue potenzialità in una visione idealistica, interessata a indicare come le cose dovrebbero essere, e sottovalutando le dinamiche, le tensioni e i significati associati alla relazione con la differenza come pratica vissuta (Colombo, Semi, 2007).

È da sottolineare come il multiculturalismo quotidiano riguardi situazioni comuni, apparentemente banali, di convivenza urbana, come dare un senso alla musica ad alto volume o all'intenso odore di cibo che si diffonde dall'abitazione del nuovo vicino, ridefinire simbolicamente lo spazio dopo che la panchina di solito utilizzata nel cortile condominiale è stata monopolizzata da un gruppo di adolescenti. Si estende a situazioni in cui la differenza può essere giocata come elemento distintivo per potenziali esclusioni o rivendicazioni di identità, oppure come giustificazione e legittimazione di situazioni asimmetriche di sfruttamento o come strumento di protezione o riconoscimento che consente resistenze o potenziali privilegi.

Muovendo da questo quadro, la ricerca presentata è interessata a indagare le pratiche di interculturalismo quotidiano che scandiscono la vita di un condominio multietnico, in cui la differenza ha assunto un significato centrale per gli attori e le attrici coinvolti, operando talvolta come vincolo, talaltra come risorsa per la definizione dei confini, favorendo faticosamente processi di inclusione, consentendo riconoscimento e, in alcuni casi, negandolo. Si è optato per l'espressione "interculturalismo quotidiano" perché ritenuta calzante per descrivere l'ambito interstiziale in cui si situa la nostra esperienza.

Un ultimo riferimento concettuale sotteso al percorso intrapreso è riconducibile all'apprendimento trasformativo (Mezirow, 2000, 2006, 2009). La teoria trasformativa rappresenta l'epistemologia di come gli adulti imparano a dare un nome alla realtà, a negoziarne i significati in modo critico e riflessivo, a capire che può essere disgiunta da ciò che si è soliti dare 
per scontato e a parlare con la propria voce, anziché accettare passivamente quelle dettate da altri (Taylor, 2009). È, questo, il processo attraverso cui il soggetto diventa consapevole di come e perché i propri assunti sono arrivati a condizionare il modo di percepire, sentire e comprendere le sue esperienze di vita e implica una riflessione critica sulle premesse distorte che ne sorreggono la struttura. I condizionamenti includono le dinamiche di potere e la loro influenza nella definizione dei processi di crescita, i canoni culturali e le strutture socioeconomiche, le ideologie e l'appartenenza etnica, le differenze di classe e di genere, le visioni del mondo e altri interessi (Mezirow, 2009).

Stereotipi, comprensioni, forme di razionalità, attenzioni selettive, atteggiamenti di negazione e minimizzazione sono strutture emergenti da un'assimilazione non riflessiva, personale e socioculturale. Di qui, l'importanza di esaminare criticamente e validare gli assunti taciti che supportano credenze, aspettative e opinioni, proprie e altrui, percepite come problematiche per (provare a) renderle più affidabili nell'orientare l'azione futura e giustificate attraverso processi dialogici, che permettano di utilizzare costruttivamente l'esperienza di altri per far sì che il significato della propria sia più attendibile e permeabile.

In questo senso, il percorso di ricerca ci ha permesso di esplicitare e formalizzare i processi di apprendimento e di autoriflessione critica che l'abitare in un condominio che nell'arco di un decennio è diventato multietnico ha consentito di intraprendere. Per esempio, il divenire maggiormente consapevoli e coscienti della nostra whiteness, rivelandone i privilegi e cercando di contribuire alla redistribuzione del potere sociale e alla riappropriazione degli spazi comuni.

\section{Percorso metodologico}

In questa cornice, è stata condotta una ricerca etnografica che ha avuto una durata di due anni e ha coinvolto 28 persone - comprese noi Autrici - residenti nel condominio. Nel corso dell' etnografia sono state svolte 26 interviste in profondità e l'osservazione di situazioni riconducibili a "incidenti critici", quali assemblee condominiali e occasioni di interazione spontanea negli spazi comuni, sia tra condomini sia tra affittuari e proprietari.

Il complesso è composto da due edifici con una corte comune e un giardino a uso esclusivo di un solo palazzo, il cui proprietario - nonostante percepisca il canone di locazione da una compagnia telefonica a cui ha permesso di installare un ripetitore sul tetto - affitta a nero cinque appartamenti a famiglie immigrate di prima generazione, rispettivamente, di origine nigeriana, libanese, tunisina e marocchina. Gli altri residenti sono inquilini regolari e/o proprietari.

La raccolta dei dati e la loro elaborazione hanno fatto parte di un processo continuo che ha visto lo svolgimento dell'elaborazione e di parte dell'analisi a ridosso della conduzione di interviste e osservazioni, seguendo la metodologia della Grounded Theory (Charmaz, 2006; Glaser, Strauss, 1967;) e utilizzando il programma di analisi dati NVivo8 ${ }^{\odot}$. L'etnografia ha rappresentato sia il metodo di ricerca sia il prodotto di differenti attribuzioni di senso (Marcus, Cushman, 1982). Il risultato è un testo polifonico, una possibile storia da raccontare plausibile, più che vera, disturbata dall'azione continua dei e delle partecipanti e orientata a sottolineare discontinuità e contraddizioni che caratterizzano i contesti di convivenza multiculturale (Bruni, 2009).

I prossimi paragrafi presentano delle etnografie raccontate per episodi, quali espedienti narrativi utili a uscire dalla pura sequenzialità temporale degli eventi, senza per questo "smembrare" i processi a questi sottostanti. Sono riportate unicamente alcune riflessioni personali, scaturite dalle osservazioni che si è cercato di condividere, più che di oggettivizzare. 


\section{La storia del condominio raccontata da Loretta}

Compro casa nel centro storico di Arezzo. Ci sono nata e ho abitato lì quando era considerato un quartiere popolare da cui tenersi alla larga. È diventata una zona borghese dove abitano i cosiddetti benestanti e benpensanti. Io voglio una casa precisa, quella in cui andavo da piccola con mio padre a trovare un suo dipendente. La vendono, e la compro. Sul retro c'è un altro edificio che ha diritto di passaggio, una corte comune e un giardino di proprietà dell'altro palazzo. Una volta ultimati i lavori per la ristrutturazione della casa, inizio a concentrarmi sui vicini. Il condominio è abitato interamente da autoctoni: c'è un anziano signore, generale in pensione, una coppia di avvocati, un giovane commercialista e un altro che lavora in un'azienda orafa. Ognuno fa i fatti suoi, si rispettano le regole condominiali più o meno implicite, come il non utilizzare in alcun modo cortile - dato che è proprietà comune, non è possibile mettere biciclette, né occupare lo spazio con stendini perché "non sarebbe decoroso".

Dopo qualche anno di "convivenza bianca" e diligente rispetto delle regole, qualcosa cambia. L'appartamento di fronte al mio viene affittato a una coppia immigrata di prima generazione di origine filippina. Se ne va il giovane italiano gentile che spacciava marjuana. Mi sento più tranquilla: ho una figlia adolescente. Avverto un certo allarmismo da parte dei condomini: porteranno connazionali clandestini; vedrai che odori terribili, che sfortuna averli nello stesso pianerottolo e proprio tu che hai restaurato da poco!

Nello stesso periodo ho bisogno di una collaboratrice domestica. La signora di origine filippina che è venuta ad abitare nel palazzo lavora presso una mia amica. Le suono e le chiedo se conosce qualcuno disponibile a lavorare a casa mia. Mi manda sua sorella: persona splendida, che ancora frequento anche se non lavora più da me. In ogni caso, i miei rapporti con $i$ Filippini - come sono chiamati dagli altri condomini - diventano stretti. Mi abituo a odori nuovi e loro ai miei rumori. Ho un cane che ulula alle campane quando è solo in casa. Hellen, la signora di origine filippina, mi dice che quel pianto porta grande sfortuna. Mi dispiace, proverò a tenerlo nelle stanze della casa più lontane dal suo appartamento, ma non ho altre soluzioni.

A Hellen e a suo marito Renato piace far festa e invitano molti amici. Utilizzano anche il cortile comune, fino ad allora considerato uno spazio intoccabile: è di tutti, quindi, nessun può usarlo. Questo agita gli animi. Gli altri condomini vengono subito a dirmi che $i$ Filippini bevono e lasciano il cortile pieno di sporcizia. Pochi giorni dopo c'è la festa di compleanno del marito di Hellen. Chiedo loro di non lasciare per terra mozziconi di sigaretta e lattine di birra. Renato mi spiega che hanno sempre pulito il giorno dopo. Io gli faccio presente che non tutti hanno pazienza e chiedo se è possibile pulire la sera stessa. Penso, medio. In realtà, sono in mezzo al guado. Il mio progressismo e ospitalità sono rassicurati da chi mette i confini.

Il condominio si trasforma in poco tempo. La casa di Hellen è frequentata da amici, ospita connazionali fintanto che non hanno trovato una qualche stabilità in termini abitativi e lavorativi. Alcuni condomini hanno paura di tutta questa gente che va e viene. Mi accorgo che c'è un altro livello di interferenza nella vita degli stranieri: un'attenzione eccessiva a ciò che fanno.

Io a poco a poco inizio a invidiarli. Perché non ho mai pensato a fare una festa nel cortile? In fondo, quel cortile lo pago in quota proporzionale ai metri quadri della casa! Inizio a pensare che la regola che impone di non utilizzare gli spazi comune sia stupida e inutile. Mi meraviglio di me. Ho aderito a una pratica omologante, Sono stata fino ad allora incapace di mettere in discussione regole condominiali che rappresentano l'esito di distorsioni sociolinguistiche e psicologiche.

Nascono altri micro-conflitti. I condomini sostengono che $i$ Filippini-non si riesce a chiamarli per nome o per cognome; tantomeno si usa l'appellativo di "signora", o "signore" - usino 
lo spazio comune come ripostiglio, ci appoggiano tutto. Io che sono amica loro, sono pregata di avvisarli. La questione mi disorienta. Inizio a condividere l'idea che gli spazi comuni non possano essere non-luoghi. Ordine e pulizia. Di li si passa, ma non si sosta. Da una parte, penso: che esagerati. Dall'altra, mi chiedo cosa accadrebbe se tutti appoggiassimo lì qualunque cosa? In fondo, quello spazio inutilizzato, ma ordinato non mi dispiace. Parlo con Hellen e le spiego cosa sta succedendo. Mi risponde che appoggia lì degli oggetti il tempo necessario a sistemarli altrove. Mi sembra una buona soluzione. Trovare uno spazio non troppo visibile e appoggiarci momentaneamente oggetti. Un altro esempio dell'uso dello spazio. Restituisco agli altri che sono d'accordo con Hellen e non ho intenzione di stare a mediare. Chiedo anche di smettere di comportarsi come se $i$ Filippini non fossero condomini come tutti gli altri, che chiedono di far entrare anche pratiche sociali diverse nella gestione degli spazi comuni.

Chiacchierando con Hellen capisco che la sua comunità è costituita da persone che perlopiù lavorano come collaboratori e collaboratrici domestiche. Vestiti, mobili, lavatrici, accessori di seconda mano si prendono, si aggiustano, si lasciano in cortile il tempo necessario per condividerli e distribuirli nella comunità che abita nelle Filippine o in quella locale. Abbiamo a che fare con un gruppo che ha problemi sociali diversi, è alle prese con la povertà e la voglia di uscirne. Hanno legami e pratiche comunitarie forti, sia nei confronti dei parenti che abitano nel luogo di origine, sia con i connazionali locali. Perché tutto questo è visto come un problema? Perché mediare? Non c'è proprio niente da mediare: c'è solo da ascoltare e imparare a riconoscere la diversità dei bisogni, senza pensare di avere più diritti solo perché bianchi.

Hellen si allarga, mette non solo degli oggetti negli spazi pubblici, ma aggiunge lo stendino con i suoi panni ad asciugare nel cortile! Mi sembra una buona idea. Ne parliamo e concordiamo che metteremo lo stendino il tempo necessario per fare asciugare i panni stesi e poi lo togliamo. Nel frattempo, un bilocale è restaurato e affittato a un personaggio aretino che si ritiene importante, della serie lei non sa chi sono io. La sua donna di servizio - come chiama la signora Marika - tiene fisso lo stendino. Hellen si lamenta: Loretta, perché lei può e noi no? Mi fa piacere. Hellen inizia a esercitare il ruolo di condomina. Andiamo a parlare con il signore lei non sa chi sono io. Hellen insiste: io vengo, ma parla tu, a me non risponde neanche. Dopo un'esplicitazione dell'accordo esistente anche il signore lei non sa chi sono io si adegua sbuffando e a malincuore.

Avverto in me un cambiamento. Il signore lei non sa chi sono io è un vicino che dovrei sentire più simile a me rispetto a Hellen, i suoi ospiti, le sue feste? Mi accorgo che la mia idea di appartenenza culturale si sta nei fatti "spacchettando". A quali pratiche di interculturalismo quotidiano voglio partecipare? Come posso modificare il regolamento del condominio in termini maggiormente inclusivi? Quali sono gli apprendimenti che sto conseguendo? Le feste, un'idea diversa di ordine e disordine, la semplicità di relazioni dove non interessa chi sei, ma come stai dentro le relazioni.

La convivenza casuale con stranieri dentro uno spazio privato, ma comune, ha creato nuove comunità che condividono tematiche diverse. Il contatto e le relazioni più o meno critiche hanno modificato i sistemi di significato. Ho avuto bisogno di fare i conti con le mie distorsioni, come il chiedermi se la mia casa rischiasse di deprezzarsi perché circondata da stranieri. Ho anche iniziato a ritenere importante la qualità delle relazioni con il vicinato: con chi convivo, quali interessi comuni possiamo trovare, come rispettare i reciproci confini?

Alla famiglia di origine filippina ne fa seguito un'altra. Vengono ad abitare nel complesso condominiale anche altri inquilini immigrati di prima generazione di origine tunisina, nigeriana, libanese, egiziana, equadoregna. C’è una famiglia di etnia igbo costituita da una madre con due figli in età scolare, una signora anziana con sua figlia, entrambe immigrate di prima generazione di origine equadoregna. Gli altri residenti sono uomini che condividono tra loro 
una qualche parentela, due fratelli, uno zio con suo nipote, tre cugini. Le famiglie di origine filippina abitano nel primo edificio del condominio e hanno un regolare contratto di locazione. I restanti condomini stranieri abitano nel secondo palazzo il cui proprietario affitta loro qualche appartamento a nero. Il condominio ha una linea di confine che simbolicamente separa la zona legale da quella illegale. I residenti bianchi sono diventati un'esigua minoranza, ma i proprietari sono convinti di detenere il potere di mantenere l'ordine.

Ricevo spesso chiamate da parte dell'amministratrice condominiale che racconta storie che assomigliano a leggende metropolitane. Mi hanno telefonato per dirmi che quei neri pisciano per le scale. Il giardino dei neri è sporco e pieno di paccottiglia, c’è anche un carrello della spesa, ma dove pensano di essere? Il ragazzo marocchino spaccia. Rispondo che l'unica cosa che ho notato più volte è che il cane della persona che ha contattato l'amministratrice è solito fare pipì ovunque e non pulisce mai le deiezioni che gli consente di fare nel giardino dei neri.

Faccio più volte presente che tutti le pagano le quote condominiali e quindi deve comportarsi come amministratrice di tutti gli inquilini. Non c'è troppo da mediare. I comportamenti, le prese di posizione, le verbalizzazioni, evidenziano un razzismo strisciante che pretende di avere un potere improprio rispetto a individui ritenuti spregevolmente stranieri, minacciosi e portatori di disordine, sicuramente di droga. Si confonde il piano legale con quello della convivenza. Dove sta la plausibilità e la fondatezza di chi afferma che $i$ neri pisciano per le scale?

Non si tratta di mediare per provare a costruire un discorso sensato. Mi trovo di fronte a persone che sono vittime della pochezza di un'esperienza di vita che non sa immaginare modi diversi di abitare un condominio. Non ci sono le condizioni per un confronto dialettico. Chi decide della validità dei punti di vista in gioco? Come si distinguono argomentazioni legittime da manifestazioni di razzismo? Mediare è un'attività necessaria, ma non sufficiente.

Lo spacciatore bianco e gentile che abitava nella casa di Hellen non era considerato disturbante l'ordine costituito. Il giovane di origine marocchina che forse fa la stessa cosa diventa un problema di sicurezza, si minaccia di chiamare le forze dell'ordine. Con quali prove, su quali evidenze?

Sparisce la mia gatta e quella di una condomina bianca che ha il coraggio, durante un'assemblea condominiale, di esplicitare il sospetto che siano $i$ Filippini a mangiare i gatti. Faccio presente che la famiglia Yape ha un gatto come animale domestico, che tra l'altro è il figlio della mia gatta sparita. Lo hanno preso anche perché ritengono che il gatto porti fortuna. Sono sempre più invisa agli altri proprietari del palazzo, ma utile all'amministratrice di condominio, la quale rispetto alla questione stranieri ha teorie-in-uso riconducibili al ma chi se ne frega e teorie-dichiarate relative a le regole sono regole e tutti dobbiamo rispettarle, perché qui funziona così.

Inizia una vera e propria "immersione". Mi trovo dentro pratiche di convivenza che ci coinvolgono. Perché le biciclette non possono stare nel cortile condominiale? Perché i cortili devono rimanere spazi vuoti, sempre, anche in estate, quando gli appartamenti sono caldi e l'ampio androne è l'unico luogo fresco? Molti condomini hanno la bicicletta e se la portano dentro. Bene. La funzionalità vince su criteri estetici quantomeno discutibili. Ci accordiamo su un disordine organizzato. Diamo ordine al nostro disordine.

Mi accorgo che, a mano a mano, si è costruita una comunità che condivide processi decisionali e pratiche di vicinato. Per esempio, il colore delle scale, i panni da stendere, le chiacchierate nel cortile, la pulizia del giardino. La signora di etnia igbo, Vivienne, si rivolge a noi quando ha bisogno di capire meglio come funzionano le questioni riguardanti la scuola dei propri figli, $\mathrm{i}$ servizi di doposcuola, le visite pediatriche. I bambini, i loro cugini e amici iniziano a girare per le case, sostano nello spazio comune, giocano. 


\section{Il giardino di Hellen e i panni stesi}

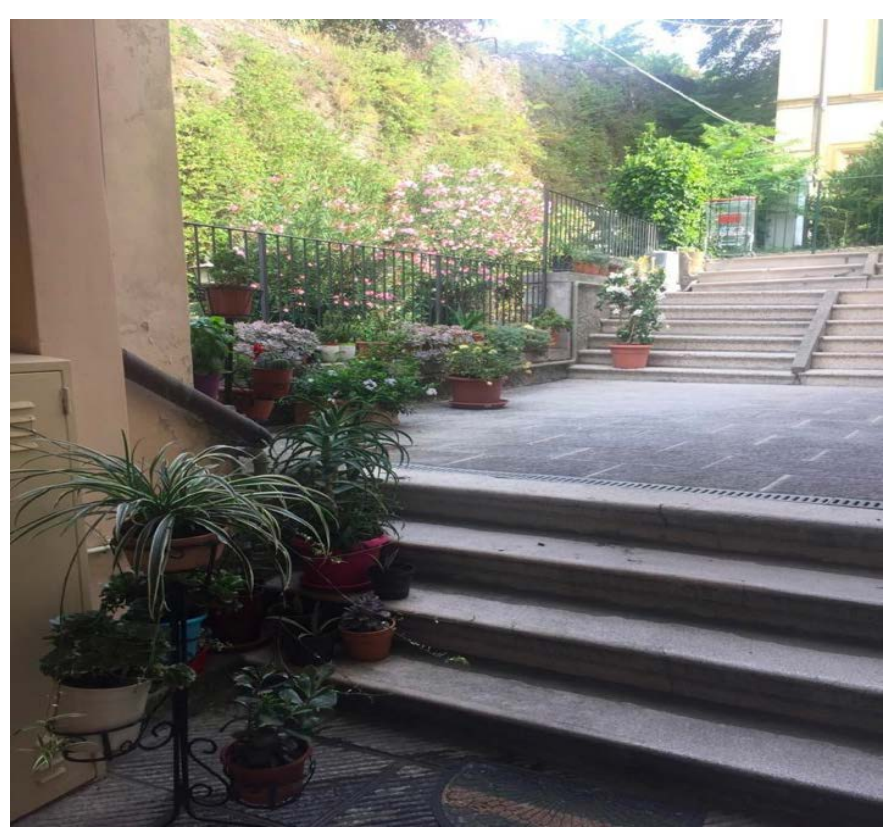

Figura 1 - Il giardino di Hellen

A Hellen piacciono molto i fiori, torna spesso dal lavoro con delle piccole piante grasse che mette subito in vasi. Queste crescono e si moltiplicano nel suo un piccolo terrazzo. Suo marito ci ha costruito una piccola serra per l'inverno. Una mattina troviamo dei vasi nel cortile comune dove c'è un muretto abbastanza brutto che diventa lo spazio dove Hellen timidamente mette le piante.

Hellen ha il pollice verde e piano piano arreda di fiori il cortile condominiale con discrezione e timore. Ci chiede spesso il permesso e con fatica accetta il fatto che quel socializzare le sue piante sia un gesto di cortesia nei confronti di tutti. Le facciamo i complimenti e la invitiamo ad allargarsi. Contribuiamo a comprare terriccio e vasi. Nel giro di pochi mesi ha costruito un giardino. Quello spazio, alquanto squallido, nudo, spoglio ma ordinato diventa uno spazio dove esplodono colori e forme. È costruito con gli scarti delle piante di una signora da cui lavora Hellen, i vasi sono semplici, per lo più di plastica. Eppure, è uno splendore. È la prima cosa che guardiamo quando torniamo a casa la sera e quando usciamo al mattino. Iniziamo a portarle dei fiori che in mano sua diventano piante rigogliose.

A un'assemblea condominiale che, data la pandemia da Covid-19, chiediamo di fare nel cortile comune, ci accorgiamo che il giardino è invisibile ai proprietari. Facciamo notare la trasformazione dello spazio e la bellezza aggiunta dalle piante di Hellen. Non per tutti il giardino rappresenta una valorizzazione di uno spazio inutilizzato. Alcuni precisano che non è opportuno che la Filippina si allarghi troppo. Quelle piante devono essere contenute. Penso che se lo avessi fatto io nessuno si sarebbe permesso di fare obiezioni. Crediamo sia il momento di mettere "i puntini sulle i". La Filippina si chiama Hellen e dato che è coetanea alla maggior parte delle persone presenti vi invitiamo a chiamarla signora Hellen. Ciò che sta facendo è un servizio a tutti $i$ 
condomini: proponiamo di ringraziarla e di contribuire alle spese, non obbligatoriamente, ma chi vuole e come crede. Il nostro posizionamento costringe tutti, almeno formalmente, ad allinearsi. Nessuno contribuisce, né apprezza esplicitamente il valore di questa crescita discreta: un giardino costruito con materiale povero, ma che la passione di Hellen ha reso vero.

Un altro episodio che vale la pena condividere è rappresentato dalla foto sottostante che documenta come Vivienne, la signora di etnia igbo, è solita stendere i panni: sull'erba e sulle siepi del giardino.

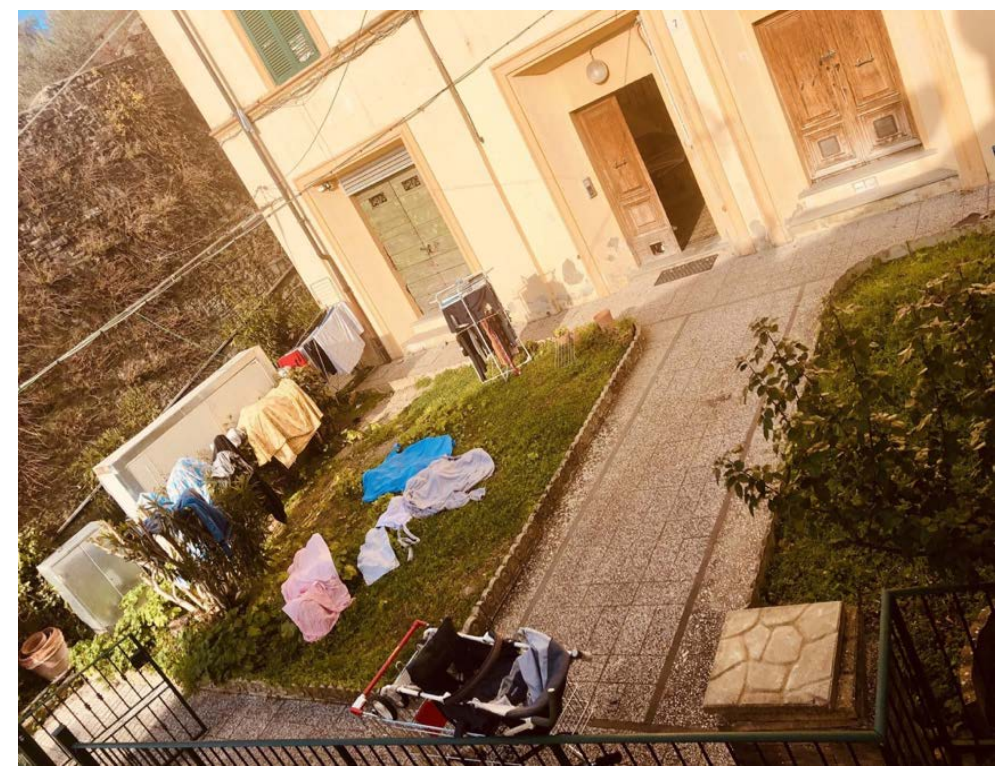

Figura 2 - I panni stesi

È uno spettacolo. La prima cosa a cui pensiamo è che anche nella nostra casa di campagna siamo solite utilizzare lo stendino, nonostante ci siano piante, siepi e alberi a volontà. Abbiamo cambiato il modo di stendere i panni, ripensando il rapporto con gli alberi, con la natura, l'idea di pulito e sporco. Ci rendiamo conto di quanto vivere tra simili rischi di essere poco creativo, non offra spunti, non stimoli l'immaginazione, rischi di farci rimanere prigionieri di ciò che abbiamo appreso troppo, tanto da pensare che ci sia un unico modo di fare. L'internazionalizzazione non è un processo confinabile nell'università. L'internazionalizzazione stimola la nostra esperienza, invitandoci a non pensarla come l'unica possibile.

\section{Considerazioni conclusive}

Abitare un contesto multietnico è stato uno dei modi per apprendere e per costruire conoscenza: le persone che abbiamo incontrato come vicini ci hanno richiesto di essere pensate, perché non appartenenti a ciò che ci era familiare. Ciò ha implicato il prendere atto di trovarci immerse in uno scenario rispetto al quale non eravamo necessariamente attrezzate, né capaci di tradurre categorie interpretative in strumenti utilizzabili per gestire situazioni dove i conflitti di interessi, di valore, i dilemmi disorientanti sono elementi caratterizzanti, all'ordine del giorno e che sollecitano ad apprendere e disapprendere. 
Città, quartieri, condomini possono essere visti come contesti di apprendimento informale. Ed è il contatto che permette di mettersi in ascolto, di porsi delle domande, di non fermarsi a prese di posizione ideologizzate, stigmatizzate e dipendenti da fonti inattendibili. Abbiamo cercato di affrontare, seppure a livello micro, il rapporto tra ordine e variazione (Mantovani, 2008a). Il quadro entro il quale ci siamo mosse è descrivibile nel cercare di analizzare come un sistema simbolico - caratterizzato da diversità e posizione polarizzate - fa i conti con la "danza dell'improvvisazione". Non ci saremo mai aspettate di convivere con tanti stranieri. Arezzo è una cittadina di provincia, per molti aspetti omologante e tendenzialmente unanimistica.

È utile riprendere gli esempi che fa Giuseppe Mantovani (2008a) di ricerche che rischiano di accreditare stereotipi etnici e di introdurre forme più o meno larvate di razzismo, come gli studi tematizzano come gruppi giustapposti o contrapposti adolescenti marocchini e adolescenti italiani, ignorando tra l'altro che molti adolescenti di origine marocchina sono anche cittadini italiani. Queste ricerche partono dal presupposto che ci siano due gruppi differenti di adolescenti, i Marocchini e gli Italiani - omogenei all'interno del gruppo, ma differenti nei confronti tra gruppi. L'Autore si chiede provocatoriamente se sia plausibile pensare che gli adolescenti marocchini siano tutti uguali (Ibidem). E gli Italiani anche, sono tutti uguali in forza del fatto di essere Italiani? E come bisogna essere per essere Marocchini? Bisogna essere Musulmani? Praticanti? E l'adolescente che ha un genitore marocchino e l'altro italiano dove lo mettiamo? Né di qua, né di là?

Si è cercato di raccogliere una delle sfide che la ricerca scientifica (Amiraux, Araya-Moreno 2014; Colombo, 2006, 2014; Mantovani, 2008b) pone, esplorando come funzionino gli scambi tra persone e come prendano forma le pratiche quotidiane di traduzione e di adeguamento che utilizzano la differenza per regolare le possibilità e le modalità di partecipazione alle azioni che hanno luogo nei contesti in cui si è inclusi.

La contrapposizione noi e loro è una costruzione sociale disfunzionale, che nel caso del nostro condominio ha generato, ad esempio, la pretesa di avere un regolamento neutro, in realtà scritto per e da comunità bianche, culturalmente ritenute omogenee, in una distribuzione del potere che non consente alle persone immigrate nemmeno di avere cittadinanza nello spazio intorno alla propria abitazione.

Altrettanto disfunzionale è pensarsi come "benefattori". Loro diventano poverini! Così è più facile accettarli: ma guarda che io li aiuto, ho dato loro vecchi cappotti, uno scatolone pieno di asciugamani, una cassettiera, ma è inaccettabile che facciano grigliate e feste nel cortile. Questo mi infastidisce.

Senza rafforzare il nostro pensiero critico, senza validare i nostri apprendimenti inconsapevoli non siamo in grado di immaginare, di ripensare in termini creativi la convivenza in una società che sarà sempre più plurale: dove incontri la differenza nel tuo quartiere e trovi ciò che è familiare all'altro capo del mondo (Geertz, 1986).

L'esperienza presentata ci ha consentito di comprendere meglio come la polarizzazione sia tra i fattori scatenanti di pensieri microradicalizzati. La volontà di rendere periferiche ogni abitudine che non appartenga al familiare e l'uso di un potere strisciante da padroni di casa mortificano chiunque condivida con noi uno spazio relazionale, prima che fisico. Ritenere le regole condominiali neutre o che il nostro senso dell'ordine sia il migliore significa pensare in termini precritici. Il pensiero precritico è quella forma di pensiero che non sa pensare diversamente, che crede che tradizioni e culture vadano difese e si immaginano guerre di valori e di principi. Il pensiero inclusivo è, invece, quel pensiero capace di entrare dentro un sistema di significato plurale, dove la diversità è fonte di generazioni di nuove pratiche funzionali a relazioni aperte a prospettive diverse, non necessariamente antitetiche. Al riguardo, ci viene in mente l'esempio 
che fa Chimamanda Ngozi Adichie a proposito del femminismo come prospettiva interpretativa: «essere femministe è come essere incinte. $\mathrm{O}$ lo si è o non lo si è. $\mathrm{O}$ credi nella piena uguaglianza tra uomini e donne, o non ci credi» (2017, trad. it. 2020, p. 10). Questo vale anche nell'affermare se siamo inclusivi, o non lo siamo.

\section{Riferimenti bibliografici}

Amiraux V., Araya-Moreno J. (2014): Religious Pluralism and Radicalization. The Missing Perspective? In L. Dawson, P. Bramadat (eds.): Religion, Radicalization and Securitization: A Canadian Perspective. Toronto: University of Toronto Press, pp. 92-120.

Amiraux V., Beauchesne P.L. (2020). Racialization and the Construction of the Problem of the Muslim Presence in Western Societies. In S. Akbarzadeh (ed.): Handbook of Political Islam. London: Routledge, pp. 363-382.

Bamberg M. (2004): Narrative Discourse and Identity. In J.C. Meister, T. Kindt, W. Schemus, M. Stein (eds.): Narratology beyond Literary Criticism. Berlin-New York: Walter de Gruyter, pp. 213-237.

Baumann G. (1996): Contesting Culture: Discourses of Identity in Multi-ethnic London. Cambridge (UK): Cambridge University Press.

Benhabib S. (2002): The Claims of Culture. Equality and Diversity in the Global Era. Princeton (NJ): Princeton University Press.

Besozzi E. (2001): L'incontro tra culture e la possibile convivenza. Studi di sociologia, 39(1), pp. 65-81. Biagioli R. (2005): Educare all'interculturalità. Teorie, modelli, esperienze scolastiche. Milano: FrancoAngeli.

Brookfield S. (1990): Using Critical Incidents to Explore Learners' Assumptions. In J. Mezirow \& Associates: Fostering Critical Reflection in Adulthood: A Guide to Transformative and Emancipatory Learning. San Francisco (CA): Jossey-Bass, pp. 177-193.

Brookfield S. (2003): Racializing the Adult Education. Harvard Educational Review, n. 73, pp. 497-523.

Bruni A. (2009): Etnografie organizzative. Rassegna Italiana di Sociologia, 50(1), pp. 185-192.

Cancellieri A. (2010): Come sopravvivere alla differenza. Etnografia dei confini sociali in un condominio multiculturale. Etnografia e ricerca qualitativa, 3(1), pp. 11-36.

Charmaz K. (2006): Constructing Grounded Theory: A Practical Guide through Qualitative Analysis. London: Sage.

Colombo E. (2006): Multiculturalismo quotidiano. Verso una definizione sociologica della differenza. Rassegna italiana di sociologia, 47(2), pp. 269-296.

Colombo E. (2014): Multiculturalismo quotidiano: luoghi e legami sociali alla prova della globalizzazione. Magma, 12(2), pp. 1-11.

Colombo E., Semi G. (a cura di) (2007): Multiculturalismo quotidiano. Le pratiche della differenza. Milano: FrancoAngeli.

Cho S., Crenshaw K.W., McCall L. (2013): Toward a Field of Intersectionality Studies: Theory, Applications, and Praxis. Signs: Journal of women in culture and society, 38(4), pp. 785-810.

Crenshaw C. (1997): Resisting Whiteness' Rhetorical Silence. Western Journal of Communication (includes Communication Reports), 61(3), pp. 253-278.

Crenshaw K. (1989): Demarginalizing the Intersection of Race and Sex: A Black Feminist Critique of Antidiscrimination Doctrine, Feminist Theory and Antiracist Politics. University of Chicago Legal Forum, 1989(1), art. 8, pp. 139-167.

Davies B., Harré R. (1990): Positioning: The Discursive Production of Selves. Journal for the Theory of Social Behaviour, 20(1), pp. 43-63.

Fabbri L. (2019): Le metodologie attive di ricerca. Che cosa hanno a che fare azione, partecipazione, apprendimento e ricerca. Educational Reflective Practices, n. 1, pp. 7-18.

Fabbri L., Amiraux V. (2020): Apprendere a vivere in una società multietnica. Educational Reflective Practices, (n.1), pp. 5-17.

Fabbri L., Melacarne C. (2020): Apprendere e disapprendere nei contesti di vita quotidiana. Il caso di un quartiere multietnico. Educational Reflective Practices, n. 1, pp. 18-54. 
Geertz C. (1986): The Uses of Diversity. Michigan Quarterly Review, n. 1, pp. 253-275.

Gherardi S. (2017): One Turn...And Now Another One: Do the Turn to Practice and the Turn to Affect Have Something in Common? Management Learning, 48(3), pp. 345-358.

Glaser B.G., Strauss A.L. (1967): The Discovery of Grounded Theory: Strategies for Qualitative Research. New Brunswick (USA)-London (UK): Aldine Transactions.

Harris A. (2009): Shifting the Boundaries of Cultural Spaces: Young People and Everyday Multiculturalism. Social Identities: Journal for the Study of Race, Nation and Culture, 15(2), pp. 187-205.

Harris C.I. (1993): Whiteness as Property. Harvard Law Review, 106(8), pp. 1707-1791.

Mantovani G. (2004): Intercultura. E possibile evitare le guerre culturali? Bologna: il Mulino.

Mantovani G. (2006): Multicultura o intercultura? Giornale italiano di psicologia, 33(2), pp. 309-315.

Mantovani G. (2008a) (a cura di): Intercultura e mediazione. Teorie ed esperienze. Roma: Carocci.

Mantovani G. (2008b): Analisi del discorso e contesto sociale. Bologna: il Mulino.

Marsick V.J., Neaman A. (2018): Adult Informal Learning. In Kahnwald N., Täubig V. (eds). Informelles Lernen: Informelles Lernen. Berlin: Springer, pp. 53-72.

Marsick V.J., Watkins K.E. (2018): Introduction to the Special Issue: An Update on Informal and Incidental Learning Theory. New Directions for Adult and Continuing Education, n. 159, pp. 9-19.

Meer N., Modood T. (2012): How does Interculturalism Contrast with Multiculturalism? Journal of Intercultural Studies, 33(2), pp. 175-196.

Mezirow J. (1991): Transformative Dimension of Adult Learning. San Francisco (CA): Jossey-Bass.

Mezirow J. (2000): Learning to Think Like an Adult. Core Concepts of Transformation Theory. In Mezirow J. \& Associates: Learning as Transformation: Critical Perspective on a Theory in Progress. San Francisco (CA): Jossey-Bass, pp. 3-34.

Mezirow J. (2004): Una teoria critica dell'apprendimento autodiretto. Trad. it. in P.G. Quaglino (a cura di): Autoformazione. Milano: Raffaello Cortina, pp. 7-23.

Mezirow J. (2006): An Overview of Transformative Learning. In P. Sutherland, J. Crowther (eds.): Lifelong Learning: Concepts and Contexts. New York: Routledge, pp. 24-38.

Mezirow J. (2009): Transformative Learning Theory. In Id., E. Taylor (eds.): Transformative Learning in Practice: Insights from Community, Workplace, and Higher Education. San Francisco (CA): JosseyBass, pp. 18-32.

Meer N., Modood T. (2012): How Does Interculturalism Contrast with Multiculturalism? Journal of Intercultural Studies, 33(2), pp. 175-196.

Ngozi Adichie C. (2016): Il pericolo di un'unica storia. Trad. it. Torino: Einaudi, 2020.

Ngozi Adichie C. (2017): Cara Ijeawele. Quindici consigli per crescere una bambina femminista. Trad. it. Torino: Einaudi, 2020.

Sandercock L. (2000): When Strangers Become Neighbours: Managing Cities of Difference. Planning Theory \& Practice, 1(1), pp. 13-30.

Sarat A. (2000): The Micropolitics of Identity/Difference: Recognition and Accommodation in Everyday Life. Daedalus, 129(4), pp. 147-168.

Silva C. (2015): Lo spazio dell'intercultura. Democrazia, diritti umani e laicità. Milano: FrancoAngeli.

St. Pierre E.A. (2015): Practices for the "New" in the New Empiricisms, the New Materialisms, and Post Qualitative Inquiry. In N. Denzin, M. Giardina (eds.): Qualitative Inquiry and the Politics of Research. Walnut Grove (CA): Left Coast Press, pp. 75-95.

Taylor E. (2009): Fostering Transformative Learning. In J. Mezirow, Id. (eds.): Transformative Learning in Practice: Insights from Community, Workplace, and Higher Education, cit., pp. 3-17.

Tarozzi M. (2015): Dall'intercultura alla giustizia sociale. Per un progetto pedagogico e politico di cittadinanza globale. Milano: FrancoAngeli.

Tomlinson J. (1999) Sentirsi a casa nel mondo. Trad. it. Milano: Feltrinelli, 2001.

Touraine A. (1997): Pourrons-nous vivre ensemble? Égaux et différents. Paris: Fayard.

Wirth L. (1938): Urbanism as a Way of Life. American Journal of Sociology, 44(1), pp. 1-24. 
